\title{
Lack of Relationship between Blood Glucose-lowering Activity of Colestimide and Serum Cholecystokinin (CCK) Concentrations in Patients with Type 2 Diabetes
}

\author{
Tatsuya Suzuki ${ }^{1,2}$, Kenzo Oba ${ }^{1,2}$, Jun Norose ${ }^{1,2}$, Hiroomi Yoshimatsu ${ }^{1,2}$, \\ Kenichi Sekimizu ${ }^{1,2}$, Shoko Futami-Suda ${ }^{1,2}$, Motoshi Ouchi ${ }^{1,2}$, Kazunari Suzuki ${ }^{1,2}$, \\ Yoshiaki Kigawa $^{3}$ and Hiroshi Nakano ${ }^{1,2}$ \\ ${ }^{1}$ Department of Functional Pathophysiology for Human Organs, Graduate School of Medicine, Nippon Medical School \\ ${ }^{2}$ Division of Geriatric Medicine, Nippon Medical School \\ ${ }^{3}$ Hanno Geriatric Hospital, Saitama
}

\begin{abstract}
Colestimide has been reported to lower blood glucose levels in patients with type 2 diabetes and hypercholesterolemia. We investigated the mechanism of the hypoglycemic activity of colestimide by examining changes in serum cholecystokinin (CCK) and insulin concentrations before and after its 2 -week oral administration. A total of seven type 2 diabetes inpatients with hypercholesterolemia received colestimide after their blood glucose levels had stabilized. We daily measured plasma glucose levels and serum lipid concentrations, calculated Body Mass Index (BMI), and determined whole-day changes in serum immunoreactive insulin (IRI) and CCK concentrations in all study subjects. We daily measured plasma glucose levels, as well as serum IRI and CCK concentrations at 10 time points for measurement. Plasma glucose levels, as well as serum IRI and CCK concentrations before and after the 2-week oral administration of colestimide were compared. The means of total cholesterol levels and BMI decreased significantly after administration. At time points for measurement (10:00 and 12 : 00), plasma glucose levels decreased significantly after administration $(P=0.026$ and $P=$ 0.009 , respectively). Diurnal changes in serum IRI and CCK concentrations were not observed after administration, except for the IRI concentration at 20: 00. The effect of colestimide on CCK may not explain the mechanism of its blood glucose-lowering activity in patients with type 2 diabetes and hypercholesterolemia.
\end{abstract}

(J Nippon Med Sch 2008; 75: 111-115)

Key words: colestimide, cholecystokinin, plasma glucose, type 2 diabetes mellitus

Correspondence to Tatsuya Suzuki, MD, PhD, Division of Internal Medicine (Department of Cardiology, Hepatology, Geriatric Medicine, and Integrated Medicine), Nippon Medical School, 1-1-5 Sendagi, Bunkyo-ku, Tokyo 113-8603, Japan

E-mail: t-suzuki@nms.ac.jp

Journal Website (http://www.nms.ac.jp/jnms/) 


\section{Introduction}

Colestimide is an anion-exchange resin that lowers cholesterol through its binding to bile acids in the intestinal tract. Some studies ${ }^{1-3}$ including ours have recently reported the blood glucose-lowering activity of colestimide in patients with type 2 diabetes. However, the mechanism by which an anionexchange resins decrease plasma glucose levels remains unknown. Watanabe et al. $^{4}$ have recently reported that the administration of cholic acid, a type of bile acids, decreases body weight and plasma glucose levels in metabolic syndrome model mice via energy expenditure by promoting intracellular thyroid hormone activation in brown adipose tissue. Colestimide is already known to alter the composition of bile acids in humans ${ }^{5}$. We have reported a modest increase in postprandial glucagonlike peptide-1 (GLP-1) levels accompanied by a decrease in postprandial glucose levels after the 1 week oral administration of colestimide ${ }^{6}$. We consider that the results from this study explain, at least in part, the blood glucose-lowering activity of colestimide because the extent of increase in GLP-1 concentrations was slight. Cholecystokinin (CCK) is also a gastrointestinal hormone capable of stimulating insulin secretion ${ }^{7.8}$. In the present study, we investigated the relationship between the blood glucose-lowering activity of colestimide and serum CCK concentrations in patients with type 2 diabetes.

\section{Patients and Methods}

The present study was conducted at our hospital in patients with type 2 diabetes $(2$ men and 5 women; mean age: $72.4 \pm 12.9$; BMI: $28.7 \pm 3.2 \mathrm{~kg} / \mathrm{m}^{2}$; duration of diabetes: $12.9 \pm 9.0$ years) who were complicated by hypercholesterolemia and whose postprandial glucose levels were poorly controlled despite a weight-maintaining diet $(25-30 \mathrm{kcal} / \mathrm{kg}$ of standard body weight) and treatment with oral hypoglycemic agents. Three patients were treated by diet alone, and four with oral hypoglycemic agents. The following oral hypoglycemic agents were administered: two subjects were treated with glimepiride, one with glibenclamide, and one with pioglitazone hydrochloride.

Colestimide (1,500 mg/day) was administered orally twice daily, before breakfast and dinner, for 2 weeks. Plasma glucose levels, as well as serum immunoreactive insulin ( IRI) and CCK concentrations were measured before and after the 2 -week oral administration of colestimide. No new drugs were added, and the doses of permitted combination drugs were not changed during the study period. Plasma glucose levels, as well as serum IRI and CCK concentrations were measured daily at 10 time points: 08 : 00 (before breakfast), 10 : 00, 12 : 00 (before lunch), $14: 00,18: 00$ (before dinner), $20: 00,00: 00,03: 00,06: 00$, and $08: 00$ in the next morning. Before the start of the study, informed consent was obtained from all subjects after a clear explanation had been provided.

Plasma glucose levels were measured by the glucose oxidase method, and serum IRI and CCK concentrations by radioimmunoassay. After admission, self-monitoring of blood glucose (SMBG) was performed by all subjects on consecutive days. The coefficient of variance for SMBG at fasting for 1 week immediately before the initiation of the study was verified to be as low as $3.0 \%$.

Data in the text, tables, and figures are expressed as means \pm SD. Student's and paired t-tests were conducted for between-group comparisons of continuous variables, and $\chi^{2}$ test for categorical variables. A value of $P<0.05$ was considered statistically significant.

\section{Results}

Baseline characteristics of study subjects are shown in Table 1. Diurnal changes in plasma glucose levels, as well as serum IRI and CCK concentrations are shown in Figure 1. At $10: 00$ and 12 : 00, plasma glucose levels decreased significantly after colestimide administration $(P=0.026, P=0.009$, respectively). No significant changes were noted in the mean IRI values after administration, except for a significant decrease $(P=0.006)$ at $20: 00$. Furthermore, no significant changes were noted in the mean CCK values, although they tended to 
Table 1 Baseline characteristics of study subjects

\begin{tabular}{lc}
\hline $\mathrm{N}$ & 7 \\
Male/female & $2 / 5$ \\
Age (years) & $72.4 \pm 12.9$ \\
Plasma fasting glucose level $(\mathrm{mg} / \mathrm{dL})$ & $123 \pm 27$ \\
HbAic (\%) & $7.8 \pm 1.1$ \\
Duration of diabetes (years) & $12.9 \pm 9.0$ \\
Body Mass Index $\left(\mathrm{kg} / \mathrm{m}^{2}\right)$ & $28.7 \pm 3.2$ \\
T-Chol (mg/dL) & $224.7 \pm 32.4$ \\
HDL-C $(\mathrm{mg} / \mathrm{dL})$ & $43.4 \pm 6.2$ \\
TG $(\mathrm{mg} / \mathrm{dL})$ & $218.3 \pm 111.5$ \\
\hline
\end{tabular}

Therapy:Diet alone or in combination with oral hypoglycemic agents.

Two subjects were treated with glimepiride, one with glibenclamide, and one with pioglitazone hydrochloride.

Values are expressed as mean \pm SD.

decrease after administration.

The mean plasma total cholesterol value decreased significantly from $224.7 \pm 32.4$ to $184.6 \pm$ $28.6 \mathrm{mg} / \mathrm{dL} \quad(P=0.001) \quad$ after administration. No significant differences were found in changes of serum high-density lipoprotein (HDL) cholesterol and triglyceride concentrations. The mean value of BMI decreased significantly from $28.7 \pm 3.2$ to $27.4 \pm 2.6$ after administration $(P=0.044)$.

\section{Discussion}

In the present study, colestimide decreased plasma glucose levels and body weight in patients with type 2 diabetes. This finding is in line with that of our previous studies ${ }^{1,6}$. However, no significant differences were noted in serum CCK concentrations before and after the 2-week oral administration of colestimide.

CCK, secreted by intestinal endocrine cells that are located mainly in the duodenum, is released into the blood stream during a meal. Many animal studies have reported that cholestyramine, an anion exchange resin, enhances the release of $\mathrm{CCK}$ in response to nutrients ${ }^{10-12}$. As demonstrated in both in vitro and in vivo studies ${ }^{10.13 .14}$, furthermore, CCK exerts a potent stimulatory action on insulin secretion. In humans, however, whether CCK has an ability to stimulate insulin secretion is controversial $^{8.15-19}$. Rushakoff and coworkers ${ }^{19}$ reported that the infusion of CCK at rates reproducing postprandial blood levels of the hormone potentiate amino acid-stimulated insulin secretion. In their study, however, the infusion of CCK alone failed to increase plasma IRI concentrations in healthy volunteers. In another study ${ }^{18}$ similar in design to the above experiments that was conducted using much lower doses of amino acids than those in the study by Rushakoff et al., i.e., doses probably leading to physiological concentrations thereof, CCK did not enhance insulin secretory response to an amino acid mixture administered intravenously that closely mimicked postprandial increments in circulating amino acid concentrations. Ahren and coworkers ${ }^{16}$ showed that a pharmacological dose of CCK given by intravenous bolus injection stimulates the release of basal and meal-related insulin. However, Reimers and coworkers $^{17}$ found that a physiological dose of CCK given by exogenous infusion does not enhance insulin release induced by the intravenous infusion of glucose and phenylalanine. Therefore, these studies suggest that CCK is not a physiological incretin hormone in humans.

The mechanism by which an anion exchange resin decreases plasma glucose levels without the changing serum concentrations of CCK and IRI remains unknown. Possible mechanisms include reduced or slowed absorption of ingested carbohydrates ${ }^{20}$. Studies in rat have shown that bile acid compounds, or cholestyramine-supplemented diets decrease ileal glucose uptake ${ }^{21}$. In addition, Watanabe et al. recently reported that administration of bile acids, especially cholic acids, induces energy expenditure by promoting intracellular thyroid hormone activation in brown adipose tissue, preventing obesity and insulin resistance in metabolic syndrome model mices. Colestimide increased fasting plasma cholic acid levels but not to a statistically significant extent ${ }^{5}$. Therefore, the improvement of metabolism with colestimide by increasing energy expenditure in skeletal muscle may partially operate via the same pathway as the supplementation with cholic acid. Farnesoid receptor X (FXR) is an orphan nuclear receptor that is activated by bile acids and has been 

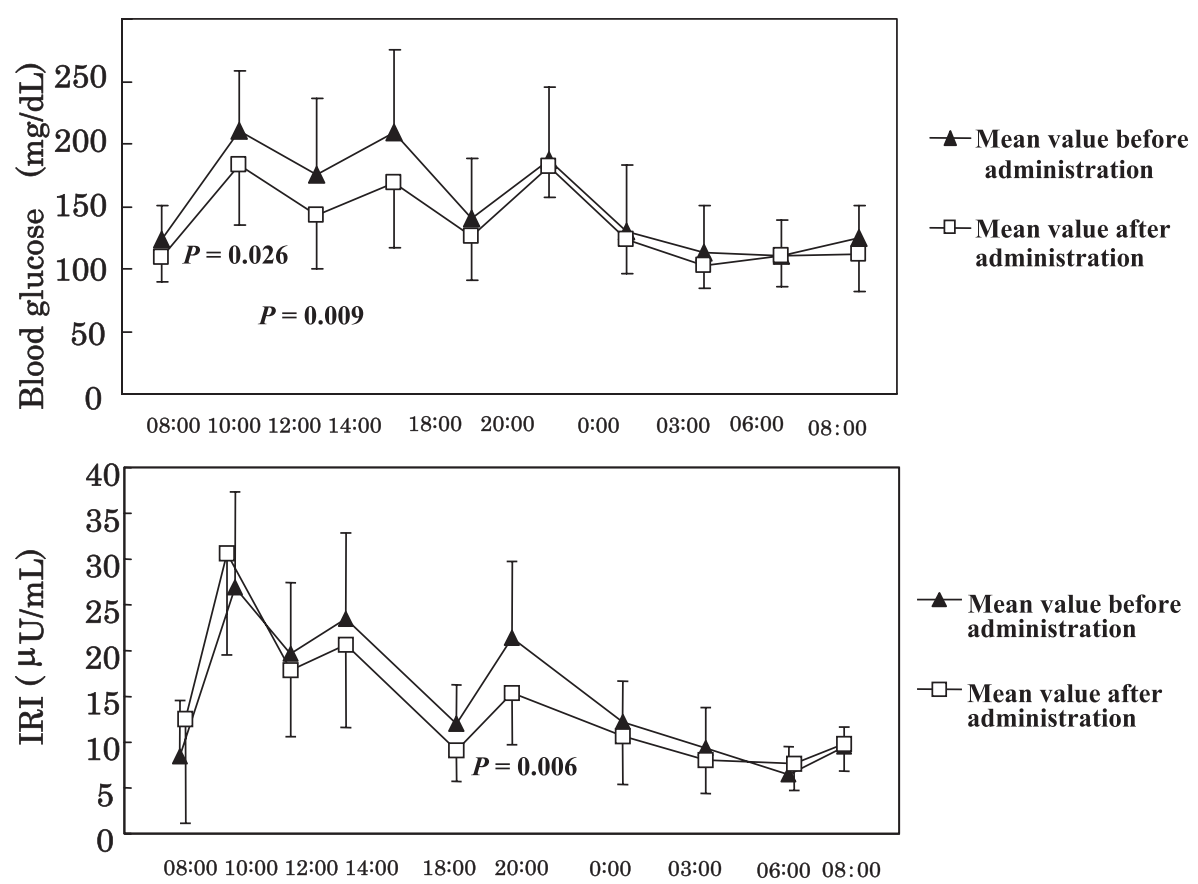

administration

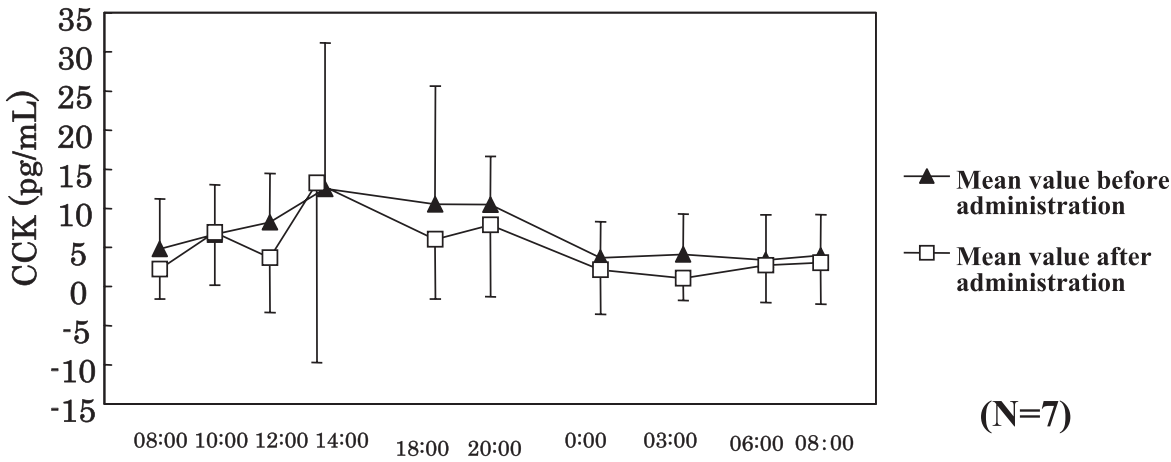

Time (hours)

Fig. 1 Diurnal changes in plasma glucose levels, as well as serum IRI and CCK concentrations before and after the 2-week oral administration of colestimide. Data are expressed as means $\pm \mathrm{SD}$.

implicated in glucose metabolism ${ }^{23}$. FXR is involved in the regulation of hepatic glucose metabolism. Therefore, colestimide may affect glucose metabolism via FXR.

The present preliminary study may also suggest the lack of a relationship between the blood glucoselowering activity of colestimide and serum CCK concentrations in patients with type 2 diabetes.

\section{References}

1. Suzuki T, Oba K, Futami S, et al:: Blood glucoselowering activity of colestimide in patients with type 2 diabetes and hypercholesterolemia: A case-control study comparing colestimide with acarbose. J
Nippon Med Sch 2006; 73: 277-284.

2. Yamakawa $\mathrm{T}$, Takano $\mathrm{T}$, Utsunomiya $\mathrm{H}$, Kadonosono K, Okamura A: Effect of colestimide therapy for glycemic control in type 2 diabetes mellitus with hypercholesterolemia. Endcr J 2007; 54: $53-58$.

3. Bloomgarden ZT: Achieving glycemic goals in type 2 diabetes. Diabetes Care 2007; 30: 174-184.

4. Watanabe M, Houten SM, Mataki C, et al.: Bile acids induce energy expenditure by promoting intracellular thyroid hormone activation. Nature 2006; 439: 484-489.

5. Kajiyama G, Tazuma S, Yamashita G, et al.: Effect of MCI-196 on biliary lipids metabolism in patients with hypercholesterolemia. J Clin Ther Med 1996; 12: 1349-1359.

6. Suzuki T, Oba K, Igari Y, et al:: Colestimide lowers plasma glucose levels and increases plasma glucagon-like peptide-1 (7-36) levels in patients with 
type 2 diabetes mellitus complicated by hyperlipidemia. J Nippon Med Sch 2007 (In press).

7. Ahren B, Holst JJ, Efendic S: Antidiabetogenic action of cholecystokinin- 8 in type 2 diabetes. J Clin Endoclinol Metab 2000; 85: 1043-1048.

8. Rushakoff RA, Goldfine ID, Beccaria LJ, Mathur A, Brand RJ, Liddle RA: Reduced postprandial cholecystokinin (CCK) secretion in patients with noninsulin-dependent diabetes mellitus: evidence for a role CCK in regulating postprandial hyperglycemia. J Clin Endocrinol Metab 1993; 76: 489-493.

9. Liddle RA, Goldfine ID, Rosen MS, Taplitz RA, Williams JA: Cholecystokinin bioactivity in human plasma. Molecular forms, responses to feeding, and relationship to gallbladder contraction. J Clin Invest 1985; 75: 1144-1152.

10. Kogire M, Gomez G, Uchida T, Ishizuka J, Greedley GH Jr, Thompson JC: Chronic effect of oral cholestyramine, a bile salt sequestrant, and exogenous cholecystokinin on insulin release in rats. Pancreas 1992; 7: 15-20.

11. Gomez G, Upp JR Jr, Lluis F, et al: Regulation of the release of cholecystokinin by bile salts in dogs and human. Gastroenterology 1988; 94: 1036-1046.

12. Gomez G, Townsend CM Jr, Maani R, Singh P, Greeley GH Jr, Thompson JC: Down regulation of pancreatic growth and gallbladder contractility by bile salts. Am J Surg 1989; 157: 20-26.

13. Karlsson S, Ahren B: Cholecystokinin-stimulated insulin secretion and protein kinase $\mathrm{C}$ in rat pancreatic islets. Acta Physiol Scand 1991; 142: 397403.

14. Ahren B, Martensson H, Nobin: A Cholecystokinin (CCK)-4 and CCK-8 stimulate islet hormones secretion in vivo in the pig. Pancreas 1988; 3: 279284.

15. Liddle RA, Gerts BJ, Kanayama S, et al.: Regulation of pancreatic endocrine function by cholecystokinin: Studies with MK-329, a nonpeptide cholecystokinin receptor antagonist. J Clin Endoclinol Metab 1990; 70: $1312-1318$.

16. Ahren B, Pettersson M, Uvans-Moberg K, Gutniak M: Efendic S Effects of cholecystokinin (CCK)-8, CCK-33, and gastric inhibitory polypeptide on basal and meal-stimulated pancreatic hormone secretion in man. Diabetes Res Clin Pract 1991; 13: 153-162.

17. Reimers J, Nauk M, Creutzfeldt W, et al.: Lack of insulinotropic effect of endogenous and exogenous cholecystokinin in man. Diabetologia 1988; 31: 271280.

18. Fieseler $\mathrm{P}$, Bridenbaugh $\mathrm{S}$, Nustede $\mathrm{R}$, et al: Physiological augmentation of amino acid-induced insulin secretion by GIP and GLP-1 but not by CCK8. Am J Physiol 1995; 268: E949-E955.

19. Rushakoff RJ, Goldfine ID, Carter JD, Liddle RA: Physiological concentrations of cholecystokinin stimulate amino acid-induced insulin release in humans. J Clin Endocrinol Metab 1987; 65: 395-401.

20. Hashim SA, Bergen SS Jr, Van Itallie TB: Experimental steatorrhea induced in man by bile acid sequestrant. Proc Soc Exp Biol Med 1961; 173175.

21. Thompson AB, Keelan M: Feeding rats diets containing choeno- or ursodeoxycholic acid or cholestyramine modifies intestinal uptake of glucose and lipids. Digestion 1987; 38: 160-170.

22. Cariou B, Duran-Sanoval D, Kuipers F, Staels B: Farnesoid receptor X: a new player in glucose metabolism? Endocrinology 2005; 146: 981-983.

(Received, October 16, 2007)

(Accepted, January 18, 2008) 